\title{
Strengths and limitations of MST radar measurements of middle-atmosphere winds
}

\author{
W. K. Hocking \\ Department of Physics and Astronomy, University of Western Ontario, Canada \\ Received: 18 November 1996 / Revised: 20 February 1997 / Accepted: 24 February 1997
}

\begin{abstract}
Radars have been used successfully for many years to measure atmospheric motions over a wide range of altitudes, from ground level up to heights of several hundred kilometres into the ionosphere. In this paper we particularly wish to concentrate on the accuracy of these measurements for winds in the middle atmosphere (i.e. $10-100-\mathrm{km}$ altitude). We begin by briefly reviewing the literature relating to comparisons between radar methods and other techniques. We demonstrate where the radar data are most and least reliable and then, in parallel with a discussion about the basic principles of the method, discuss why these different regimes have the different accuracies and precisions they do. This discussion is used to highlight the strengths and weaknesses of radar methods. Issues like radar volume, aspect sensitivity, gravity wave effects and scatterer intermittency in producing wind biases, and the degree by which the intermittent generation of scatterers at quasi-random points in space could skew the radar measurements, are all considered. We also investigate the possibility that MF radar techniques can be contaminated by E-region scatter to heights as low as $92-95-\mathrm{km}$ altitude (i.e. up to $8-10 \mathrm{~km}$ below the ionospheric peak echo). Within all these comments, however, we also recognize that radar methods still represent powerful techniques which have an important future at all levels of the atmosphere.
\end{abstract}

\section{Introduction}

Radars used for atmospheric wind measurements cover a broad range of styles and applications, from dish-like systems as used for meteorological work (e.g. Atlas, 1990) and ionospheric work (e.g. Mathews, 1984) to phased array systems (e.g. Woodman and Guillen, 1974; Röttger et al., 1978; Fukao et al., 1985a,b) and simpler spaced-antenna systems (e.g. Vincent et al., 1987). In addition to a multiplicity of antenna types, there are different hardware arrangements, which involve various types of pulse compression (e.g. Farley, 1985) and integration procedures. We will not dwell on these aspects, but will try broadly to classify different radars according to their methods of data analysis, since as a rule it is the different techniques which are most important in our discussions, rather than the technical details of the radars being used.

Several different techniques are applied in radar measurements of atmospheric winds. These include the Doppler method (Woodman, 1985; Zrnic, 1979; Hocking, 1989), the spaced-antenna method (e.g. Briggs, 1984; Hocking et al., 1989), and variations on the spaced-antenna method, which act in an interferometric manner (e.g. Adams et al., 1985; Röttger and Ierkic, 1985; Röttger et al., 1990). Figure 1 demonstrates schematically some of these techniques. We will not dwell in detail on the methods, save to give a brief overview. The reader is referred to the references given for more detailed discussions. However, in brief the methods can be summarized as follows.

The Doppler method uses a large (often steerable) antenna to form a narrow beam, and then directs a large fraction of the available transmitter power along this "pencil beam". The radio waves scatter from irregularities within the beam, and are scattered to a receiving array which collects the signal. In many cases the receiver array is the same one as the transmitter array, and this arrangement is called a monostatic radar (Fig. 1a). The returning signal is Doppler shifted relative to the transmitted one, due to the motions of the scatterers, and the radar then measures this Doppler shift. Typical values for the Doppler shift are in the range $0.01-10 \mathrm{~Hz}$. The value of the Doppler shift is then used to determine the mean radial velocity of the scatterers within the beam, and by using combinations of radial velocities obtained with different beam pointing directions, it is possible to make measurements of the nett motion of the atmospheric scatterers.

The spaced-antenna method generally utilizes different antennas for transmission and reception, and rather 


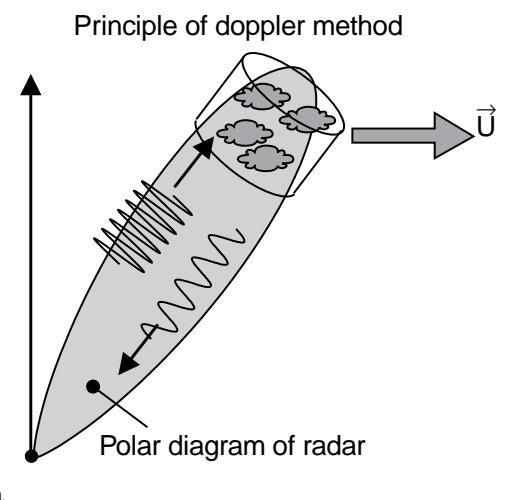

a

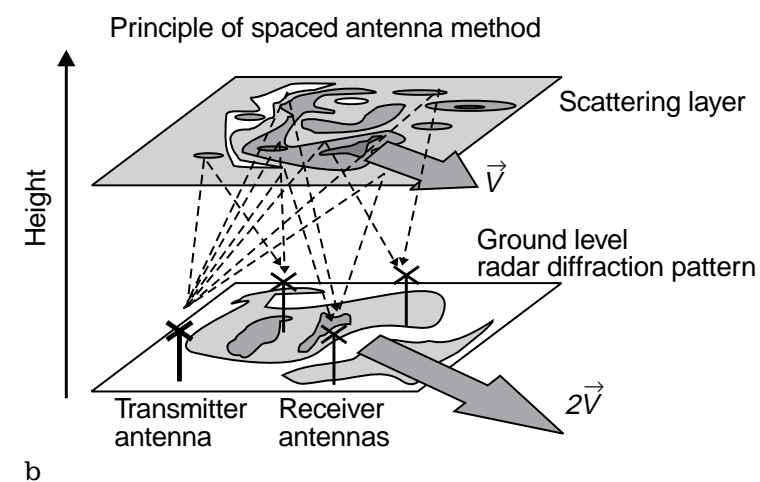

Principle of interferometry

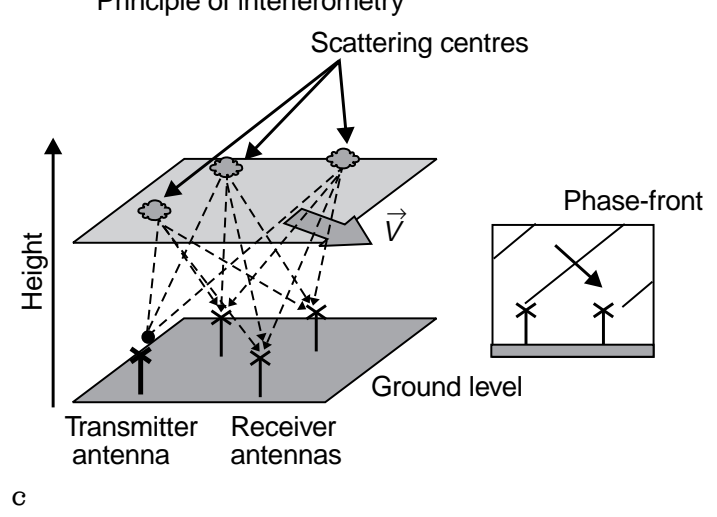

Fig. 1a-c. Schematic diagram representing the main principles applied in radar techniques. a demonstrates the Doppler method, b shows the spaced-antenna method and $\mathbf{c}$ shows the interferometric principle. In a, the Doppler shift of the returning radiation relative to that transmitted is indicated, and this shift is the basis of the method. In b, the scattering distribution over the sky at one altitude is shown, together with the diffraction pattern on the ground. Note that the broken lines represent just a few of the many "ray paths" which can exist; the radiation propagating along these paths eventually interferes at the ground to give the resultant diffraction pattern. In $\mathrm{c}$, the effective ray paths associated with just a few scatterers are shown. The inset to the right shows incoming phase fronts from a single scatterer in two dimensions, illustrating that a single phase front arrives at different antennas at different times. The delay between phase fronts arriving at different antennas is used to determine the original location of the scatterers

than directly measuring the scatterer's radial velocities, it measures the drift of the diffraction pattern of the scatterers across the ground (Fig. 1b). At least three groups of antennas, spatially separated over the ground, are used to record the backscattered signals, and cross-correlation functions calculated between pairs of receivers are used to infer the drift speed of the diffraction pattern. The drift speed of the scatterers themselves are then found as one half of the drift speed of the diffraction pattern (e.g. Briggs, 1992), and the direction of drift is the same for both the scatterers and the diffraction pattern. Although the method seems in principle to be different to the Doppler method, Briggs (1980) has shown that they are actually very similar in basic principle. However, a major difference is the fact that the spaced-antenna method often utilizes small groups of antennas (for either transmission or reception or even both), with correspondingly larger beam widths, and we will see later that this broader beam-width can have important implications for the method. The spaced-antenna method also tends to make more use of scatterers closer to overhead of the radar, and this point will also prove important later on.

Interferometric methods use a combination of these procedures, but are limited by the requirement that the scattering region must contain only a few discrete scatterers. By using complex correlation functions determined between spaced receiving antennas, it is possible to find the directions of discrete scatterers within the sky, and then determine Doppler shifts associated with each individual scatterer (Fig. 1c). By combining many such measurements of scatterer locations and Doppler shifts, it is then possible to determine mean velocities as a function of height which closely approximate the mean values over the radar. However, the method breaks down if there are too many scatterers within the beam; for example, in the case of volume scatter (many scatterers distributed almost continuously in space), it is not possible to unambiguously identify discrete scattering entities.

Extensive tests of these different procedures have been applied at many altitudes, but we will begin by considering comparisons in the lowest altitude of the atmosphere (i.e. especially in the troposphere and lower stratosphere). Parts a and b of Fig. 2 illustrate nicely comparisons between the Doppler method applied with narrow-beam radars and radiosonde measurements, and it is clear that in general the agreement between the methods is quite good. These show comparisons from Crane (1980) and Hocking (1997). Other detailed comparisons (not shown) have been presented by many authors, including particularly careful studies by Weber and Wuertz (1990) and Astin and Thomas (1992). In addition, Röttger and Vincent (1978), Larsen and Röttger (1982) and Vincent et al. (1987) have demonstrated the accuracy of the spaced-antenna method in the troposhere. There is little question that Doppler and spaced-antenna techniques work quite well in the troposphere, where the scatterers are generated relatively continuously in height and time, so we may conclude that there is nothing intrinsically wrong with the basic theory of the methods. However, some of the specifics of implementation can lead to problems in certain situations, and these special cases will be one subject of this 


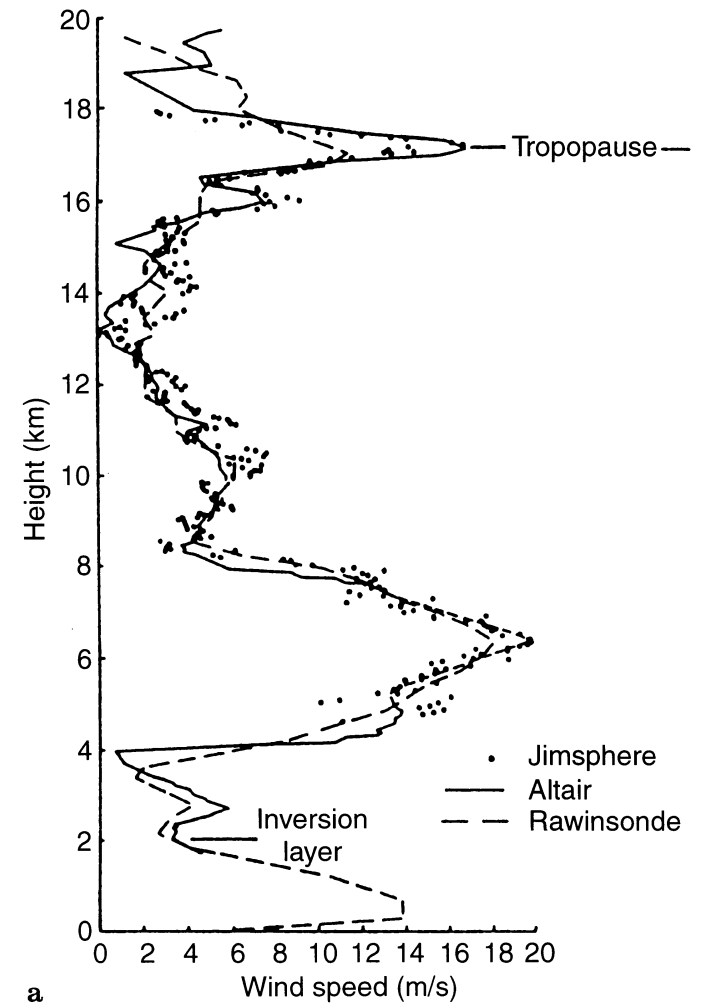

Fig. 2a, b. Illustration of comparisons between the Doppler method and radiosonde measurements, showing a comparisons of magnitudes of winds using a UHF radar (Crane, 1980) and b comparisons of magnitudes directions between VHF radar winds and radiosondes (Hocking, 1997). Note that in the second case the slope of the line of

article. These special cases can be especially important in the upper atmosphere (above $60-\mathrm{km}$ altitude), and it is to this region that we now turn.

The importance of determining the accuracy and reliability of radar winds in the mesosphere has always been crucial, but is even more so now that new satellite techniques [especially WINDII (e.g. Shepherd et al., 1993; Gault et al., 1996; McLandress et al., 1996) and HRDI (Hays et al., 1993; Burrage et al., 1996)] have begun producing large quantities of data. Studies incorporating data from different systems like radars and satellites will need to be carried out more frequently in the future, and knowledge about the relative accuracies of each system will be important in such comparisons. As seen, radar comparisons with other techniques generally show good agreement in the lower atmosphere, but as the altitude of the scatterers reaches mesospheric heights, we note that the level of agreement degrades somewhat; comparisons of radar techniques at these heights are not always as good as in the lower atmosphere. Cases reporting moderate to good agreement include those due to Stubbs (1973), Stubbs and Vincent (1973), Vincent et al. (1977), Vincent and
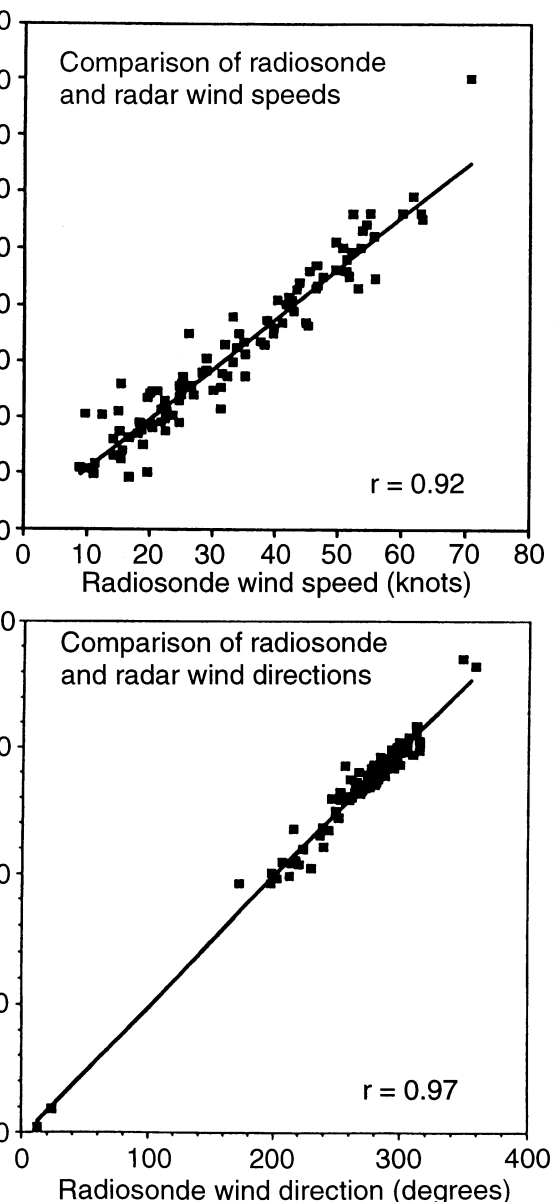

best fit for the magnitudes is not quite 1.0, due to the effect scatterer anisotropy. This effect is usually corrected for, but we have chosen not to do so in this case. See the text for a more extensive discussion of scatterer anisotropy

Stubbs (1977) and Phillips et al. (1994), but cases of poor agreement also exist, e.g. Hines et al. (1993). Even the cases of apparently "good" agreement are not as well matched as tropospheric comparisons [e.g. Stubbs (1973), especially above $90 \mathrm{~km}$ ], although it is also true that some of the cases of "bad" agreement have been to some extent explained (e.g. Turek et al., 1995). There are good understandable reasons for this great degree of variability and discrepancy, particularly in relation to the fact that the beam width of a radar in terms of physical width (kilometers) becomes quite large at large ranges, and also the fact that the entities producing the backscatter become more intermittent in space and time at the higher heights. In addition, there is often a greater degree of inherent wind variability at these upper heights, where wave activity becomes more vigorous in the higher regions (e.g. Hines, 1960). Thus comparisons on short time-scales invariably show variability, and longer-term averages are required to show better agreement. For example, Hocking and Thayaparan (1997) have shown fairly good comparisons of semidiurnal tidal amplitudes and phases using meteor and MF techniques, but greater scatter on scales of minutes. 
Indeed (and for similar reasons), comparisons between any pairs of methods (radar or otherwise) often become worse at these higher altitudes. It is this level of agreement of different techniques, especially with regard to radar methods, which forms a major thrust of this article. However, in order to set things into perspective, we will begin not by looking at radar weaknesses, but rather at their strengths.

\section{Strengths of radar techniques}

The greatest strengths of radar techniques are almost certainly their ability to provide wind measurements during conditions of both clear air and cloud, and in all sorts of different geographic and atmospheric environments. The data acquired by radar techniques are generally among the most continuous of any procedure. The ability to run radars unattended for long periods of time, and often during both day and night, is also a key strength of the technique.

Another important advantage of radar systems is their resolution ability. Because a radar can determine the range to a scattering region, it has important advantages over other techniques in this regard. For example, passive optical techniques which rely on local atmospheric generation of the source radiation which they utilize often suffer from the fact that the exact height of the source region is unknown. In addition, it can be true that the source region can be spread over a wide band in height, producing a significant smearing of wind measurements.

The spatial accuracy of a radar system is often defined by the radar volume, which is the volume defined by the beam width and effective pulse length of the radars used. (In this context, "effective pulse length" refers to the radar resolution, i.e. the true transmitted pulse length divided by 2 , to allow for the fact that the transmitted pulse travels up but the reflected pulse down). To first order, this volume is usually a cylindrical region, with the axis of circular symmetry directed along the radar beam (see Fig. 1a). The length of the cylinder is the pulse length, and the radius is taken as the half-power halfwidth of the radar beam multiplied by the range under study. The angular beam width of the radar will not change, so that as one increases in altitude the physical width of the beam in the direction transverse to its pointing direction will increase, resulting in a worsening resolution at higher altitudes. For example, a radar with a beam full width of perhaps $5^{\circ}$ has a diameter for its radar volume at $10 \mathrm{~km}$ range of $1 \mathrm{~km}$, whilst the transverse extent covered by the beam at an altitude of $90 \mathrm{~km}$ can be typically $10 \mathrm{~km}$ across. Broader beams can correspond to horizontal extents of several tens of kilometers at these higher heights, and we will see later that these larger horizontal beam-width scales at the higher heights can be important, especially when considered in connection with the intermittency of the scattering process. With respect to pulse lengths, some radars such as VHF radars can achieve pulse lengths down to several hundred meters and better, whereas other radar systems such as MF (medium-frequency) radars are restricted to pulse lengths of the order of several kilometers.

It should be emphasized that the sorts of spatial and temporal resolutions which can be obtained with radar techniques are certainly as good as, if not better, than many optical techniques, but it must nevertheless be borne in mind that they are not perfect. Lidars, for example, have superior spatial and temporal resolution (e.g. Chanin and Hauchecorne, 1981; Gardner and Voelz, 1985), but have only recently developed the ability to measure winds, and are limited in their coverage temporally, being restricted to conditions of no cloud and night-time studies. On the other hand, radars are certainly superior to many other groundbased optical techniques in their spatial resolution, and likewise have better spatial resolution than satellite optical methods. Limb-scanning optical instruments, for example, integrate along a path of several hundred kilometers in length in their velocity determinations.

Having now pointed out the good spatial resolution capabilities of radars, we have to also temper these comments with a warning; we will see later that the fact that resolution is not perfect can still lead to some problems. For example, we will see that the issue of the volume of the atmosphere which returns scattered signals to the radar combined with the possibility of intermittent generation of scatterers, both temporally and spatially, can lead to problems with the data interpretation.

The prices of radars can also be an advantage, in that it is often possible to develop small radar systems capable of continuous unattended operation for measurement of atmospheric winds at costs of US\$ 100000. of course, more expensive systems also exist; a wide range of options are available.

At this point we will say little more about the advantage of radars - we consider that the strengths are to a large extent self-evident. We will concentrate rather on a critical review of the limitations of radar methods, although it is important to emphasize that whilst we intend to be quite honest about these problems, most other techniques (including both optical and satellite) suffer from problems which are at least comparable, if not even worse.

\section{Potential limitations of radar techniques}

In the following sections, we will discuss some important aspects relating to the application of radar techniques. Perhaps the main issue of concern is the fact that these methods usually (with the exception of meteor radars) depend critically on the generation of small-scale inhomogeneities within the atmosphere by turbulent and small-scale processes. It is this fact which leads to many of the potential problems in bias which will be discussed in this paper. Whilst the generation of these irregularities are certainly absolutely necessary for radars to work, the fact that the irregularities can be generated intermittently in space and time has the potential for leading to biases. We will return to this 
issue of the nature of the scatterers in due course, but before doing so we also need to look at some other special limiting factors of radar techniques.

\subsection{Vertical motions}

As our first major point, we begin by noting the possibility that some of the methods used in radar techniques can be contaminated by the effects of vertical motions. This effect is not unique to radar studies, but we will discuss it in this context.

The major problem which arises when using Doppler methods is that the radial velocities measured contain contributions from both the horizontal and vertical components of the true wind. In order to measure the horizontal winds, it is necessary to make some assumptions about the vertical motions. In some cases it is assumed that the vertical wind is zero, but since beam tilts can be as little as $10^{\circ}$ off-zenith, this can be a dangerous assumption. Even vertical winds which are less than $5 \%$ in magnitude of the horizontal winds can produce contamination. Alternative procedures include using a vertical beam to make measurements of the vertical wind, and incorporating these measurements into the determinations of horizontal velocities. However, generally the vertical winds and the off-vertical radial components are not measured in the same physical volume of space, and errors can arise due to these spatial differences. In addition, there can be errors in vertical velocity measurements due to the fact that scattering entities can have small tilts which skew the region of scatter away from directly overhead; this issue has been discussed by, amongst others, Larsen and Röttger (1991) and Larsen et al. (1992).

As seen, Doppler radars are especially vulnerable to the effects of vertical winds because of the small zenithal beam pointing angles often employed in radar experiments. It may seem that the spaced-antenna method is not so vulnerable to this effect, but this is not true either. Briggs (1980) has shown how the drift of a diffraction pattern across the ground can be envisaged as the "beating" effect of signals scattered (with appropriate Doppler shifts) from different points in the beam, so that vertical motions can also produce errors in horizontal velocity determinations in this case too. However, we should emphasize that the contamination in this case is less severe than with Doppler radars, since it requires rather circumstances - namely a situation in which the vertical velocity is upward on one side of the beam and downward on the other.

The idea that even the spaced-antenna method could be occasionally contaminated by vertical velocities was first seriously considered by Royrvik (1983), with subsequent modeling work presented by a variety of authors. For example, Kudeki et al. (1993) proposed a wave-induced fluctuation model and showed that systematic wind estimation errors should be expected to result from spatial variation in the vertical component of the perturbation wind field associated with gravity waves. These effects could be especially important in waves with short periods and short horizontal wavelengths, which have relatively large vertical velocities. The fluctuation biases may be non-minor in instantaneous velocity estimates but will average to zero mean in a long-term temporal average, suggesting that estimates of tidal and longer-period waves should be relatively accurate. This explanation was further substantiated and supported by more detailed numerical simulation results presented by Surucu et al. (1995). Only isotropic scattering was considered by Kudeki et al. (1993), but the numerical simulations were carried out for both isotropic and non-isotropic scatterers with different sets of gravity wave parameters by Surucu et al. (1995). (We will consider the results of anisotropic scattering more fully shortly.) Surucu et al. (1995) found that individual horizontal SA wind estimates are very well predicted by the model of Kudeki et al. (1993) as long as the gravity wave has a horizontal wavelength larger than the horizontal dimension of the MF radar scattering volume.

Thus we see that while vertical velocities can affect radar measurements of horizontal winds, the effects are most serious on time-scales of a few minutes, and generally are not of great concern for studies of motions with temporal scales of hours and more. Even at the shorter time-scales the radars can still produce measurements, but greater care is needed in recognizing and removing the effects of vertical motions.

\subsection{Scatterer anisotropy}

Another potential problem with radar measurements is the fact that the scatterers themselves are on the average anisotropic. In other words, the scattered signal magnitude varies as a function of the angle at which the beam is pointed, with generally stronger scatter from overhead and increasingly weaker scatter as the angle of the beam is tilted further and further away from vertical. This can affect measurements of the magnitudes of the winds

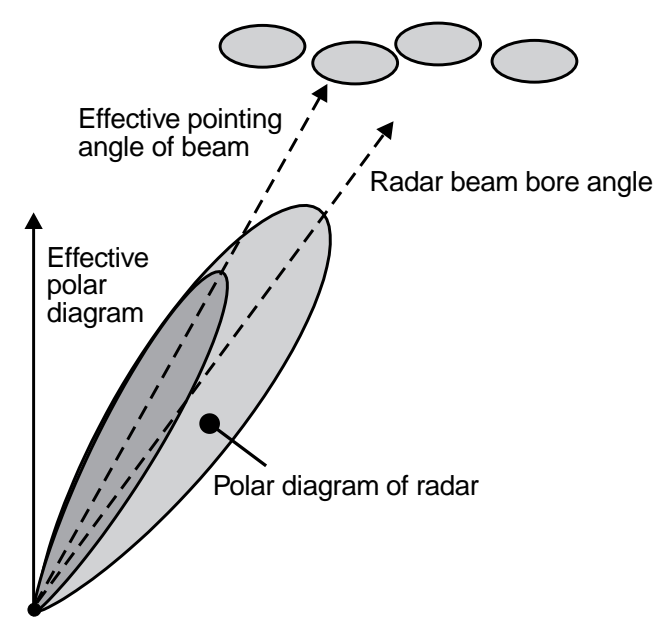

Fig. 3. Schematic diagram showing how anisotropic scatterers alter the effective beam pattern for a Doppler radar. The bulk of the radio scatter comes back through the "effective beam", rather than the true beam, due to the asymmetry introduced by the scatterers 
because it can result in a bias of the effective look-angle of the radar beam. This effect is illustrated in Fig. 3, where it can be seen that scatter is preferentially produced from angles closer to overhead than the true bore angle of the radar beam (also see Röttger, 1981). Thus when horizontal velocities are estimated via a relation like $v_{H}=v_{\text {rad }} / \sin (\theta)$, and $\theta$ is chosen to be the bore angle of the beam, then the calculated horizontal wind speed is an underestimate. Figure $2 b$ shows an example of this type of bias, where it can be seen that the slope of the graph of radiosonde wind magnitudes vs radar wind magnitudes is not unity. We emphasize that is possible to correct for these effects (see shortly); our point here is simply to recognize that the problem exists. Note that we should also emphasize that while these scatterers are often modeled as ellipsoids (e.g. Hocking, 1987), they are in reality twisted and contorted stringlike structures which are simply elliptical in a statistical sense (e.g. Hocking and Hamza, 1997).

Figure 4 illustrates the different types of scattering shapes which can be statistically representative of the scattering medium. These may involve steps, or possibly even peaks and dips, in refractive index, which have a broadly ellipsoidal shape on average. They are represented by analytical functions such as those shown in the figure and generally produce backscattered power as a function of angle which follows the form

$\mathbf{P}(\theta) \propto \exp \left\{-\left(\theta^{2}\right) /\left(\theta_{s}^{2}\right)\right\}$.

There are other possible models, but this is at least the most common model representation of the way in which power falls off.

It is possible to measure the degree of anisotropy by a variety of techniques (Hocking, 1987; Lesicar and Hocking, 1992; Lesicar et al., 1994) and extensive studies of this parameter have been made by many authors. The most complete set is probably that due to Lesicar et al. (1994), who performed studies at frequencies of around $2 \mathrm{MHz}$ at polar, mid-latitude, and equatorial stations. Similar studies have been made in the mid-latitude troposphere and stratosphere at VHF frequencies (around $50 \mathrm{MHz}$ ) by Tsuda et al. (1986) and Hocking et al. (1990). The effect of this anisotropy is to produce underestimates of the mean wind, and (as already noted) Fig. 2b shows an example. It is relatively easy to correct for the effects of this anisotropy to determine horizontal winds (e.g. Hocking, 1989). Indeed spaced-antenna wind measurements are not even sensitive to the degree of anisotropy, and measure wind speeds without any bias due to this effect.

However, there is one extreme case of anisotropy which distinguishes itself from all others because of its unique nature, and this is the phenomenon of so-called "specular reflection". In this process the backscattered radiation acts as if it were scattered by smooth mirrorlike reflectors (possibly with some degree of undulation) in the upper atmosphere. This has been observed on occasions and, while a phenomenon of significant intrinsic interest, it can also be important because it can lead to biases in wind measurements. Specular reflectors are not by any means the main form of scatterer, but they have had a substantial history of discussion in the literature, and it would be remiss of us not to discuss them here. They reveal themselves as scatterers with very, very small values of $\theta_{s}$ (typically less than about $2^{\circ}$ or so); we will now consider these very special reflectors in some more detail.

\subsection{Specular reflections}

The nature of specular reflectors has been the subject of substantial debate over many years, and various models have been proposed to explain them. A few authors have questioned the existence of these unusual entities (e.g. Woodman and Chu, 1989), but a substantial number of researchers believe that these phenomena really are something unique and distinct from turbulence. Recent balloon measurements (e.g. Luce et al., 1995, and references therein) have given extra credence to their viability, at least in the stratosphere. Figure 5 is a fairly convincing example of the spectrum produced by a
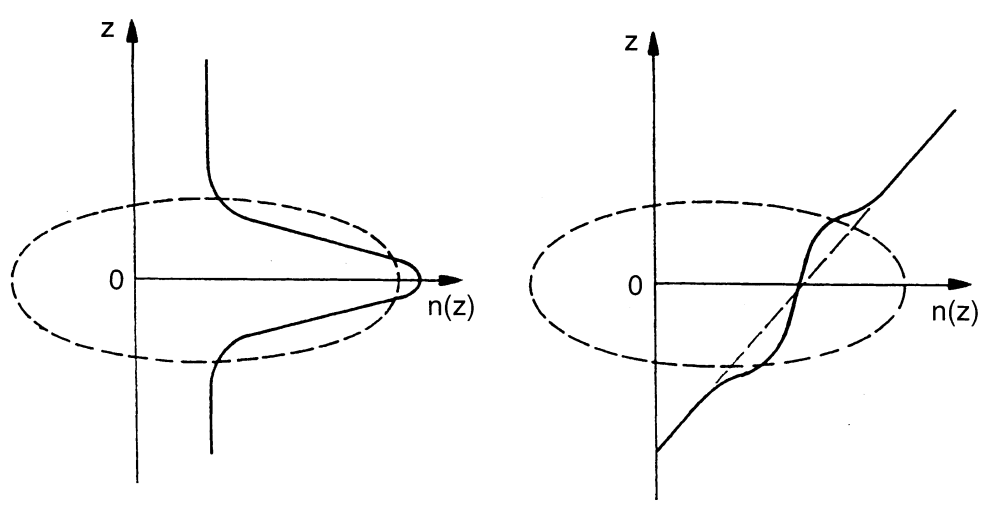

$$
\begin{gathered}
n(z) \alpha \\
c+e^{-\left[\left(x^{2}+y^{2}\right) / L^{2}+z^{2} / h^{2}\right]}
\end{gathered}
$$

a

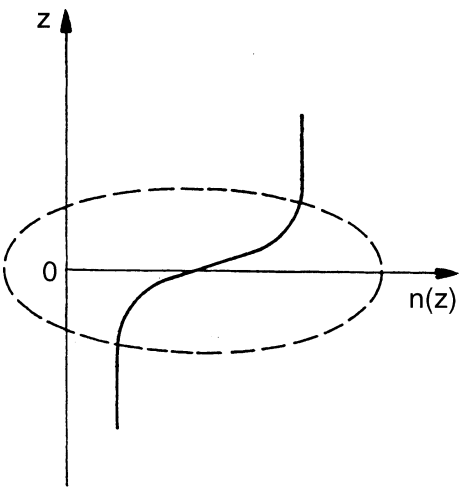

$$
c+e^{-\left[\left(x^{2}+y^{2}\right) / L^{2}\right]} \frac{1}{h} \int_{0}^{z} e^{-\zeta^{2} / h^{2}} d \zeta
$$

Fig. 4a-c. More detailed representations of the average shapes of radio-wave scatterers (from Hocking, 1987) 


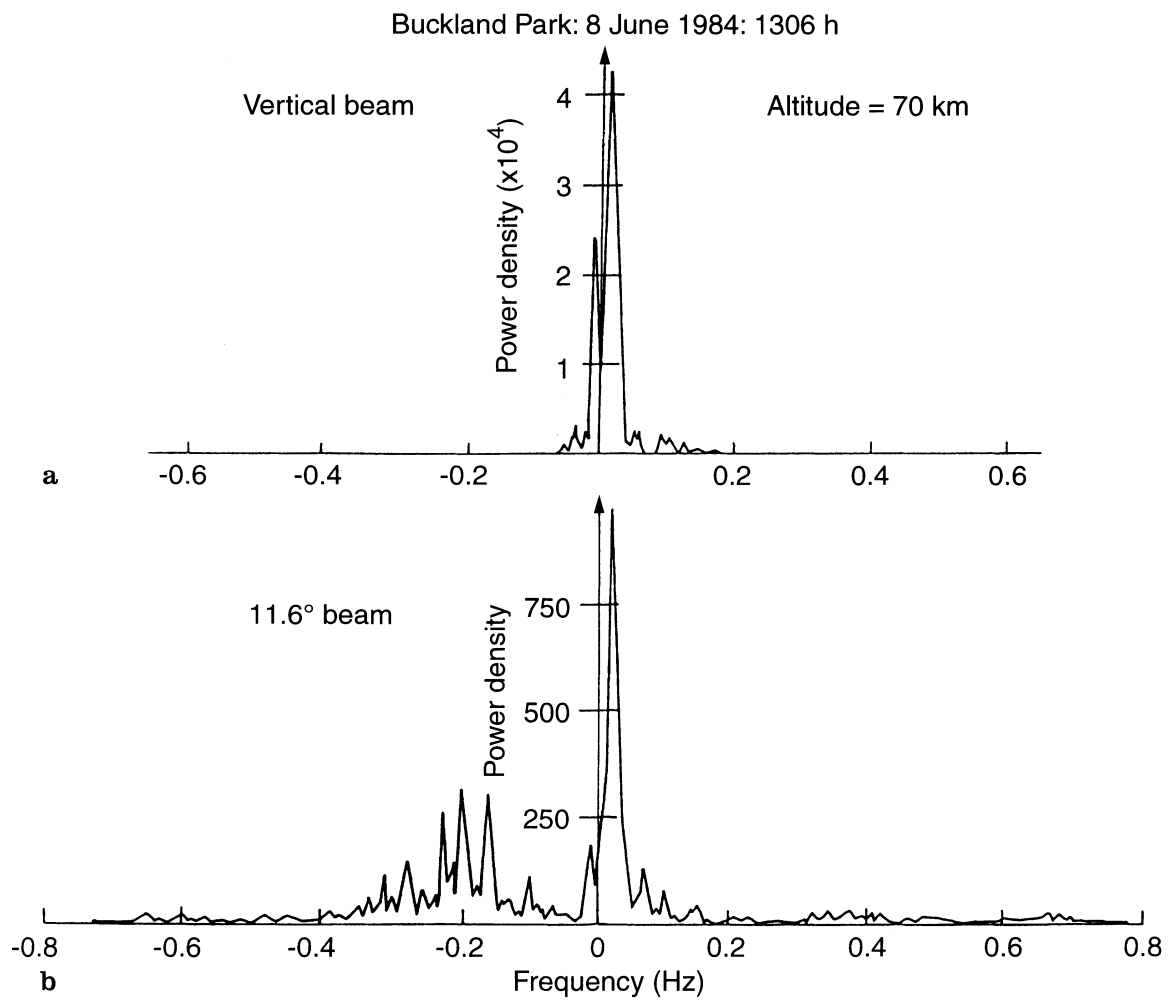

Fig. 5a, b. This figure, from Hocking (1987), demonstrates the temporally simultaneous occurrence of specular reflectors and turbulence scatter in the atmosphere, a vertical radar beam, $\mathbf{b}$ off-vertical radar beam specular reflector; it shows the simultaneous occurrence of specular reflectors and turbulence scatter in the atmosphere. The diagram comes from Hocking (1987) and shows a radar experiment which demonstrates that specular reflectors and turbulence scatterers can occur simultaneously in time, although it is generally believed that they are spatially separated. In the experiment used to produce Fig. 5, two radar beams were used simultaneously, one pointed vertically (producing Fig. 5a) and one pointing off-vertically (Fig. 5b). The large spike is due to a very strong specular reflector immediately overhead, and although it should not have been evident on the off-vertical beam, the reflector was so strong that its reflected signal was able to leak in through the sidelobes of the off-vertical beam. The smaller cluster of spectral points at around $-0.2 \mathrm{~Hz}$ in Fig. $5 \mathrm{~b}$ are genuine turbulent scatter received by the off-vertical beam. Models for specular reflectors have been discussed by Bolgiano (1968), Hocking et al. (1991), and references therein.

Specular reflectors have been of great interest in the history of radar studies, because it has been claimed that they can produce a number of biases in wind measurement, especially when the spaced-antenna method is employed.

The procedure by which this was envisaged to apply is as follows. It was assumed that gravity waves might perturb the specular reflectors to give them long smooth oscillations, with scales matching the gravity waves. In some cases these undulations were considered due to gravity waves distorting already existing layers, whereas in other models it was assumed that the waves might have short vertical wavelengths (a few tens of meters) and actually cause the radio-wave reflections themselves. We shall see shortly that this second model has been largely refuted, but for now we will not concern ourselves with the details. Rather, we will simply note that these models assumed that the gravitywave-induced oscillations were supposed to move with the wave, and therefore with the phase velocity of the waves. Thus, its was argued, the spaced-antenna method would measure the phase velocities of gravity waves, whereas say the Doppler technique would measure the true winds because an off-vertical Doppler beam is not sensitive to specular reflectors. Hines et al. (1993) incorporated these notions, together with a model of wave-wave critical-level interactions and wave-filtering (which he called "scavenging"), to show how the interactions within a wave-field superposed on a mean wind could lead to preferred senses of direction of phase velocities of gravity waves at different heights under different circumstances. This model was then used to infer predictions about the expected sorts of "winds" which might be measured by the spacedantenna technique if it was indeed sensitive to the phase speeds of gravity waves.

However, for these reflectors to be important requires that the specular reflectors have horizontal extents of many kilometers and vertical depths of less than 30 or $40 \mathrm{~m}$ at $2 \mathrm{MHz}$ and less than about 1-2 $\mathrm{m}$ at VHF. The likelihood of these scatterers existing with such huge extents, and the possible effects that they will have on radar measurements, have been discussed by Hocking et al. (1989) and Surucu et al. (1995). Hocking et al. (1989) have shown that if such specular reflectors exist they are generally intermittent, and this will destroy 
their usefulness for measurement of any winds. As a result, they will generally be rejected by traditional spaced-antenna algorithms, and as a result will not skew the measurements. It has also been argued that they are very rare (e.g. Lesicar et al., 1994), and their predominance in the literature is simply an indication of the curiosity value associated with them, rather than a measure of their frequency of occurrence. However, in addition to the arguments in Hocking et al. (1989), Surucu et al. (1995) have modeled the effects of including such distorted reflectors using computer techniques and have concluded that even if such reflectors exist they will not produce biases in long-term average winds. They may produce errors on short time-scales (a few minutes) if they exist, but Hocking et al. (1989) have indicated that even this is likely to be rare.

The most complete models for these specular reflectors are those due to Bolgiano (1968) and Hocking et al. (1991). As already noted, earlier models argued that the specular reflectors could actually be due to gravity waves themselves (e.g. Hines and Rao, 1968), but Hocking et al. (1989) have argued that this would require waves which would in fact be heavily viscously damped and therefore, in order to produce appropriate vertical wavelengths, would have significant off-vertical tilts. This effect has not been observed. Thus we would argue that these proposed mechanisms are not important in affecting gravity wave measurements. There is, however, another argument which invokes gravity wave contamination of the wind measurements produced by radars, and this proposal has much greater viability. It relates to the distribution of scatterers in space and time.

\subsection{Temporal and spatial intermittency of the scattering irregularities}

It seems fairly well established that turbulence, especially at these upper altitudes, can be and is likely to be intermittent (e.g. Hocking, 1991, 1996; Hines, 1991). These patches of turbulence have been called "white caps", and various numerical simulations of this "patchiness" have been presented (e.g. Desaubies and Smith, 1982; Hocking, 1991; Fairall et al., 1991). Some high-resolution confirmation of this "white caps" model has been provided by Sica and Thorsley (1996). As a consequence, patches of turbulence (and therefore radio-wave scatterers) occur at only isolated locations within the radar volume, thereby allowing the possibility that the "wind" measured by a radar at any instant may not represent an average over the whole volume, but may in fact simply represent the winds at one or two discrete points within the volume. The issue then becomes: How well do the measurements at one or two discrete regions in a large radar volume represent the mean wind in that region? Figure 6 illustrates the types of problems involved, and the answer to this question now needs to be discussed.

If we begin by assuming that wind measurements made at any point do truly represent the wind at that point in space and time, even though the measurement is biased to that region, then we might expect that over a reasonably long-term average the average of all these measurements should still produce a reasonable representation of the real wind. But can we assume that any such measurement really does represent the wind even at the location of the scatterer? And even if this is so, can we assume that the averaging process is unbiased?

We shall tackle the first question first. Naively, one might expect that the wind measurements really do reflect the real wind, but there have been arguments presented which disagree with this supposition. It has in fact been proposed that the measured wind velocities may be more commensurate with the phase velocity of a generating gravity wave than with the winds actually generated in that region of space (the latter would be a combination of the external wind field plus the effect of the winds due to the gravity wave itself). This model assumes that the radar "sees" the envelope of the turbulent patch and measures the drift of that envelope. Is this true? In fact it is not; in reality the radar "sees" the scatterers inside the envelope (the eddies with scales of the order of half a wavelength). Croft (1972) has extensively discussed this potential conflict between the roles of the motion of the envelope and the motions of the internal scatterers, and despite some exceptions concluded that in general Doppler radars really do measure the motions insider the envelope of the turbulence, rather than the motions of the envelope itself. Since these internal eddies are driven by the local wind, which will be due to both local winds and the gravity wave's own velocity field, their motions are truly representative of the winds at this point in time. Thus we recognize that our original "naive" picture of the radar measurements was in fact correct, and in most circumstances the radar does measure a real wind. This is also the generally accepted view within the radar community, i.e. that the radar measures the true wind because it measures the eddies moving inside the turbulent region. Nevertheless, we now recognize that this conclusion is not as trivial as we might have at first thought, and some degree of caution still needs to be exercised in this regard.

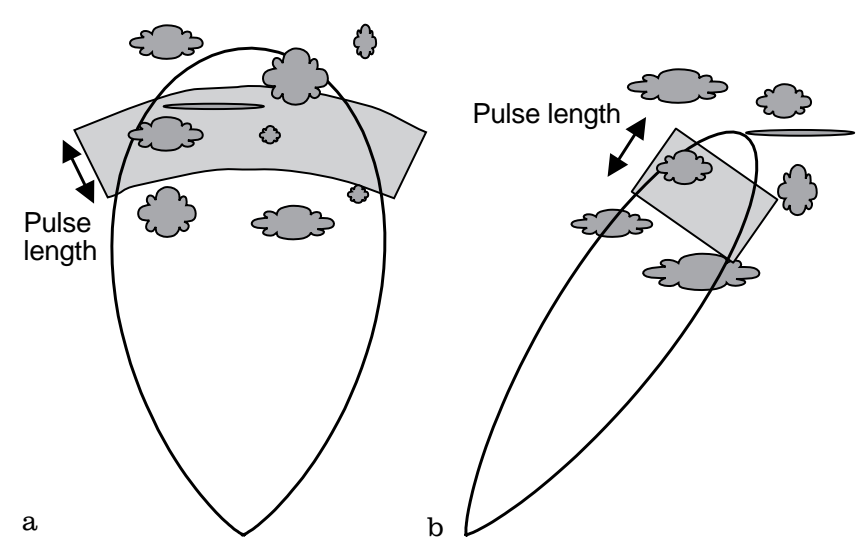

Fig. 6a, b. Schematic representations of the typical distribution of scattering centers in the atmosphere. The spatial intermittency within the beams are especially highlighted 
We now turn to the second question, viz., can we assume that the averaging process is unbiased? We can begin by recognizing that there are occasions during which the averaging process certainly is biased. For example Collis et al. (1992), and May et al. (1988), showed examples in which, because the turbulence occurs at preferred positions within the radar beam, biases of wind measurement relative to the true mean can be produced. In particular Collis et al. (1992) show how a single turbulent layer can produce artificial wind shears in radar data. This possibility was also recognized by Hocking (1983). But could such biases be more widespread? Hines (1991) and Nastrom and Van Zandt $(1994,1996)$ have independently proposed that this is a real possibility. Their rationale begins by recognizing that gravity waves may preferentially generate turbulence at certain phases of their cycles, and so as a consequence the wind speeds measured by the radar may be preferentially selective. For example, suppose (as is often likely) that a gravity wave breaks at phases of the wave during which the total wind velocity (background plus wave velocities) is in the same general direction as the phase speed of the wave. If one then averages over an ensemble of such measurements, it is possible that one may produce a bias towards measurements which are broadly in the direction of the wave speed simply because one is only measuring wind speeds at points in space and time which have directions commensurate with the original phase speed of the wave.

Experimental evidence of a similar effect has already appeared with regard to vertical motions. Nastrom and Van Zandt (1994) have used experimental data to show that preferential selection of downward velocities in the atmosphere by radars in the presence of gravity waves is a real effect, even in the troposphere. Hoppe and Fritts (1995) have shown similar effects in the mesopause region of the atmosphere. This downward bias, although slight, arises because scatterers are generated preferentially during downward motion rather than other motions within the gravity wave.

Hines' (1991) original proposal was, however, in terms of biases in horizontal motions, rather than vertical velocities. One might expect that there should be no such effect if the gravity wave field were isotropic, since on average all these effects should cancel out. However, the effect can become important if the gravity wave field is anisotropic; then one can produce horizontal biases in the mean wind due to the effects described by Hines (1991). Experimental evidence in support of this model has been presented by Nastrom and Van Zandt (1996) using tropospheric and stratospheric data, although the effect is only slight. Hines has proposed that the effect is more dramatic in the upper atmosphere, where wind variability is much greater. If the root-mean-square horizontal velocities of the gravity waves are of the order of $20-30 \mathrm{~m} \mathrm{~s}^{-1}$ at these heights, with a substantial bias of phase propagation in one direction, then errors in the horizontal velocities of the order of a few $\mathrm{m} \mathrm{s}^{-1}$ can result.

Thus we have to admit that this is a very real (even likely) possibility. It is potentially the most serious error of any which we have discussed, and whilst errors of a few $\mathrm{m} \mathrm{s}^{-1}$ may not always be of concern, they can be important on occasions. For example, monthly mean winds are often only of the order of a few $\mathrm{m} \mathrm{s}^{-1}$, so the type of error discussed here could be important for such determinations. The relative importance of this effect is still yet to be properly determined, but it definitely needs to be investigated further.

\subsection{E-region contamination}

There is one other important source of wind bias which has not been discussed very extensively, if at all, in the literature. This relates exclusively to $\mathrm{MF}$ and $\mathrm{HF}$ radar measurements above $90 \mathrm{~km}$. It is well known that the fact that the E region overlies the upper middle atmosphere can lead to contamination of some of the data around $95 \mathrm{~km}$ due to group retarded E-region echoes (e.g. Namboothiri et al., 1993). However, there is an additional effect which is not so widely known or understood, but which can at times affect wind measurements even down to $90 \mathrm{~km}$. In this section we wish to describe this effect. It is an effect related to the finite pulse width of the transmitter signal and the fact that the E-region echoes can be a hundred or even a thousand times stronger in amplitude than the D-region echoes.

When a radar pulse transmits up into the atmosphere and returns to the receiver, it peaks some time after it reaches the receiver (Fig. 7a). The delay is only a few tens of microseconds, but can be equivalent to typically 8-10 km in range or more for a typical MF radar receiver. When the range markers on a system are calibrated they are set to the peak of the echo, and not the instant of arrival of the pulse. Thus, as shown in Fig. 7b, the front end of the E-region echo can co-exist with the peak of say a $95-\mathrm{km}$ echo. However, the Eregion echo is often much stronger than the $95-\mathrm{km}$ echo; the reflection coefficient for an echo in the range 92$95 \mathrm{~km}$ might be typically $10^{-3}$ (e.g. Hocking, 1987), whilst the E-region echo from say $100-\mathrm{km}$ true altitude is totally reflected. Even accounting for absorption, the effective reflection coefficient for the E-region echo could easily be $10^{-1}$ or more. If the radar pulse after passing through the receiver has a half-amplitude halfwidth of $3 \mathrm{~km}$, so that its amplitude varies with the form $\exp \left\{-[\ln 2]\left(z-z_{\mathrm{o}}\right)^{2} /\left(3^{2}\right)\right\}$, then at a distance of $8 \mathrm{~km}$ from the peak its amplitude is reduced by about 100 times compared to the peak. Hence for an E-region echo from say $103 \mathrm{~km}$, the front end of the E-region echo at $95 \mathrm{~km}$ will have an effective reflection coefficient of typically $10^{-3}$ : comparable to the actual atmospheric signal itself. If the peak of the E-region echo comes from an effective altitude delay of $100 \mathrm{~km}$, its leading edge could even dominate over a 92-km echo. Thus the front end of the E-region echo can easily exceed the true atmospheric echoes in the region $92-95 \mathrm{~km}$. As a result, there may be times of day, especially when the E-region echo is low in altitude and strong (as can happen around midday, especially in summer), that signals from the E region could contaminate the real signals reflected from 


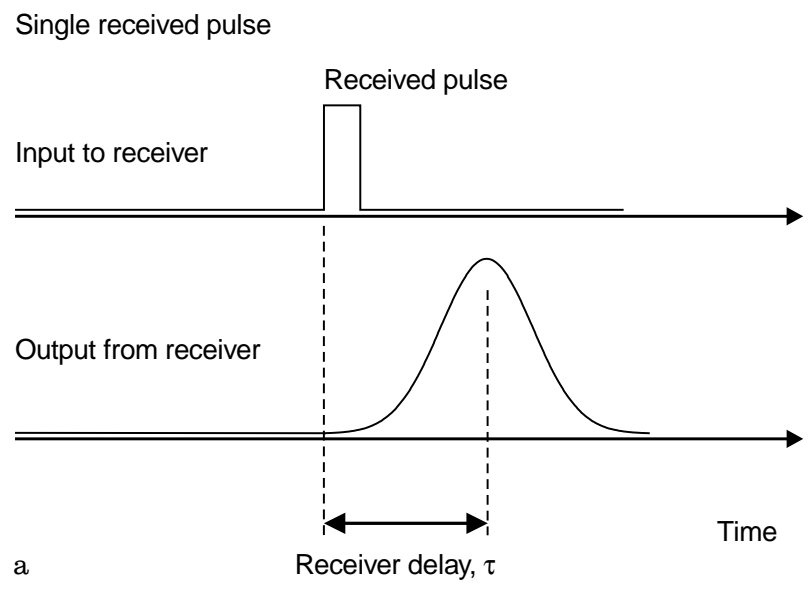

Pulses received from $95 \mathrm{~km}$ and E-region

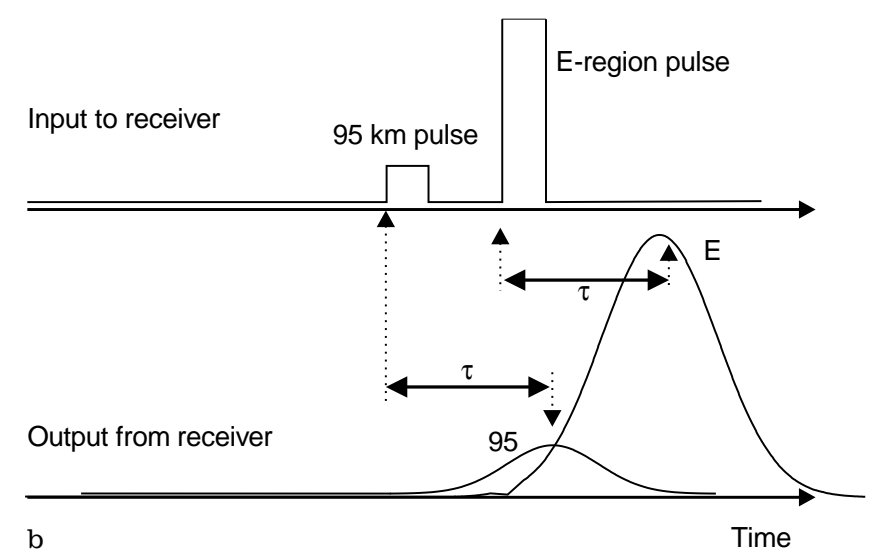

Fig. 7a. Schematic diagram showing the effect of passing a pulse through a receiver. Note especially the final Gaussian shape and delayed peak. b Schematic diagram showing the output of a typical receiver with two incident pulses, the second slightly delayed from the first. The first pulse to arrive also has a much weaker amplitude. See text for details

above $92 \mathrm{~km}$. As a result, "winds" determined at these heights and these times by MF techniques could be a mixture of E-region drifts and true winds. It has yet to be determined just how important this effect could be, but preliminary experiments performed by this author have shown that at times there can be a significant crosscorrelation coefficient between the raw fading data at 92 and $100 \mathrm{~km}-$ an indication that this effect does happen at times. The effect is unlikely to be important at nighttime, but certainly could be important around midday on some days, thereby skewing tidal estimates and daily mean winds. We should also emphasize that our schematic diagrams in Fig. 7 assume that the pulse returning form the $\mathrm{E}$ region is a square pulse. This is highly idealized; in reality, even the transmitted pulse will have a Gaussian shape, and this could spread the discussed effect to even lower heights (although probably not below $90 \mathrm{~km}$ ).

\section{Discussion}

All the preceding effects become amplified as range increases. The physical beam width becomes larger, while the degree of intermittency tends to increase and is much higher in the middle atmosphere than in the troposphere. There is also the issue of the nature of the generation of the scatterers, which seems to be intimately related to the level of gravity wave activity at the altitude of the scatterers. In particular, in the middle atmosphere the waves reach large amplitudes and also develop a level of anisotropy in preferred directions which can lead to some degree of bias.

We need at this point to reiterate that radar workers have been aware of these problems, or at least most of these problems, for many years, and, in general, have been able either to correct for them or recognize when they should not be using their data. In a sense this is something of a self-critical review of radar methods, and if optical or rocket techniques were subject to a similar degree of inquiry equivalent errors and biases would also be found in those procedures. In particular, one of the major weaknesses of rocket and optical techniques is the fact that they tend to be made only intermittently in time. Rocket measurements are usually dependent on campaigns which can last for only a week or so, and which occur at irregular intervals scattered throughout the year. Thus they have the possibility of not being a true representative of the overall statistical situation, because each rocket profile represents only a single snapshot through the atmosphere. Optical techniques are particularly impeded by the occurrence of sunlight which clearly restricts them to night-time observation, and also the occurrence of clouds which can prevent measurements over a substantial fraction of the year. Optical techniques are also subject to the fact that the height of scatter or the height of the source of the radiation is an unknown quantity. Thus we ask the reader to bear in mind that this attempt to present a somewhat self-critical review of the limitations of radars should not be perceived as a reason to rule out the viability of radar techniques. There is no question that radars have contributed enormous amounts of information to our understanding of upper atmosphere winds and will continue to do so in the future. However, this said, it is certainly true that one must bear in mind the fact that there are some inherent problems which in the main become worse at higher altitudes.

\section{Conclusions}

The strengths and limitations of radar measurements have been considered. Strengths include continuity of data, day and night-time operation, and the ability to see through clouds, as well as (often) superior spatial resolution. Various limitations were also discussed, as well as some earlier proposals which claimed that radar data could often give false winds. Some of the earlier concepts have been largely disproved, but some prob- 
lems have been recognized. The significance of contamination by vertical winds when beam zenithal tilts of $10^{\circ}$ or $15^{\circ}$ are used, and the importance of the temporal and spatial intermittency of the scatterers, have been highlighted, as has the fact that radar winds can be biased to some degree in the presence of an anisotropic gravity wave field. For example, if the gravity waves propagate predominantly in the eastward direction, some bias of winds towards the east can occur. The level of this error is likely to be substantially less than the RMS gravity wave amplitude, and so would not be expected to be much more than perhaps $5 \mathrm{~m} \mathrm{~s}^{-1}$ or so; however, future experiments to examine this effect are needed. Finally, we have shown that because the E-region echoes at MF are much stronger than D-region echoes, there is a possibility that the leading edge of the E-region echoes could contaminate MF data down to about $92 \mathrm{~km}$ or so. This possibility needs to be further investigated in the future.

However, we finally recognize that radar techniques are still a powerful and important technique for upperatmosphere wind measurements, and will continue to be a major tool in the future.

Acknowledgements. Topical Editor F. Vial thanks U.-P. Hoppe for his help in evaluating this paper.

\section{References}

Adams, G. W., D. P. Edwards, and J. W. Brosnahan, The imaging Doppler interferometer; Data analysis, Radio Sci., 20, 1481$1492,1985$.

Astin, I., and L. Thomas, The accuracy and precision of radar wind measurements. in STEP handbook, Proc. Fifth Workshop Technical and Scientific Aspects of MST Radar, Ed. B. Edwards, pp $15-20,1992$.

Atlas, D. (ed), Radar in meteorology: Battan memorial and 40th anniversary, radar meteorology conference, Am. Meteorol. Soc., Boston, 1990.

Bolgiano, R., Jr. The general theory of turbulence - turbulence in the atmosphere, in Winds and turbulence in the stratosphere, mesosphere and ionosphere, Ed. K. Rawer, North-holland, Amsterdam, pp 371-400, 1968.

Briggs, B. H., Radar observations of atmospheric winds and turbulence: comparison of techniques, J. Atmos. Terr. Phys., 42, 823-833, 1980.

Briggs, B. H., The analysis of spaced sensor records by correlation techniques, Handb. MAP, 13, 166-186, 1984.

Briggs, B. H., Radar measurements of aspect sensitivity of atmospheric scatterers using spaced-antenna correlation techniques, J. Atmos. Terr. Phys., 54, 153-165, 1992.

Burrage, M. D., W. R. Skinner, D. A. Gell, P. B. Hays, A. R. Marshall, D. A. Ortland, A. H. Manson, S. J. Franke, D. C. Fritts, P. Hoffman, C. McLandress, R. Niciejewski, F. J. Schmidlin, G. G. Shepherd, W. Singer, T. Tsuda, and R. A. Vincent, Validation of mesosphere and lower thermosphere winds from the high-resolution Doppler imager on UARS, $J$. Geophys. Res., 101, 10365-10392, 1996.

Chanin, M. L., and A. Hauchecorne, Lidar observation of gravity and tidal waves in the stratosphere and mesosphere, J. Geophys. Res., 86, 9715-9721, 1981.

Collis, P. N., M. T. Rietveld, J. Röttger, and W. K. Hocking, Turbulence scattering layers in the middle-mesosphere observed by the Eiscat $224 \mathrm{MHz}$ radar, Radio Sci., 27, 97-107, 1992.

Crane, R. K., Radar measurements of wind at Kwajalein, Radio Sci., 15, 383-394, 1980.
Croft, T. A., Sky-wave backscatter: a means of observing our environment at great distances, Rev. Geophys. Space Phys., 10, 73-155, 1972.

Desaubies, Y., and W. K. Smith, Statistics of Richardson number and instability in oceanic internal waves, J. Phys. Oceanogr., 12, 1245-1259, 1982.

Fairall, C. W., A. B. White, and D. W. Thomson, A stochastic model of gravity-wave-induced clear-air turbulence, J. Atmos. Sci., 48, 1771-1790, 1991.

Farley, D. T., On-line data processing techniques for MST radars, Radio Sci., 20, 1177-1184, 1985.

Fukao, S., T. Sato, T. Tsuda, S. Kato, K. Wakasugi, and T. Makihira, The MU radar with an active phased array system 1. Antenna and power amplifiers, Radio Sci., 20, 1155-1168, $1985 \mathrm{a}$.

Fukao, S., T. Tsuda, T. Sato, S. Kato, K. Wakasugi, and T. Makihira, The MU radar with an active phased array system 2. In-house equipment, Radio Sci., 20, 1169-1176, 1985 b.

Gardner, C. S., and D. G. Voelz, Lidar measurements of gravity wave saturation effects in the sodium layer, Geophys. Res. Lett., 12, 765-768, 1985.

Gault, W. A., G. Thuillier, G. G. Shepherd, S. P. Zhang, R. H. Wiens, W. E. Ward, C. Tai, B. H. Solheim, Y. J. Rochon, C. McLandress, C. Lathuillere, V. Fauliot, M. Herse, C. H. Hersom, R. Gattinger, L. Bourg, M. D. Burrage, S. J. Franke, G. Hernandez, A. Manson, R. Niciejewski, and R. A. Vincent, Validation of $\mathrm{O}(\mathrm{S})$ wind measurements by WINDII: The wind imaging interferometer on UARS, J. Geophys. Res., 101, 1040510430, 1996.

Hays, P. B., V. J. Abreu, M. E. Dobbs, D. A. Gell, H. J. Grassl, and W. R. Skinner, The high-resolution Doppler imager on the upper-atmosphere research satellite, J. Geophys. Res., 98, 10713-10723, 1993.

Hines, C. O., Internal atmospheric gravity waves of ionospheric heights, Can. J. Phys., 38, 1441-1481, 1960.

Hines, C. O., The saturation of gravity waves in the middle atmosphere. Part III. Formation of the turbopause and of turbulent layers beneath it, J. Atmos. Sci., 48, 1380-1385, 1991.

Hines, C. O., and R. Rhagava Rao, Validity of three station methods of determining ionospheric motions, J. Atmos. Terr. Phys., 30, 979-993, 1968.

Hines, C. O., G. W. Adams, J. W. Brosnahan, F. T. Djuth, M. P. Sulzer, C. A. Tepley, and J. S. Van Baelen, Multi-instrument observations of mesospheric motions over Arecibo: comparisons and interpretations, J. Atmos. Terr. Phys., 55, 241-287, 1993.

Hocking, W. K., On the extraction of atmospheric turbulence parameters from radar backscatter Doppler spectra-I: theory, $J$. Atmos. Terr. Phys., 45, 89-102, 1983.

Hocking, W. K., Radar studies of small-scale structure in the upper middle atmosphere and lower ionosphere, Adv Space Res., 7, 327-338, 1987.

Hocking, W. K., Target parameter estimation, Middle Atmosphere Program Handbook, SCOSTEP Secretariat, Dept. of Electr. Computer Eng., Univ of Illinois, Urbana, IL 61801, USA, 30, 228-268, 1989.

Hocking, W. K., The effects of middle-atmosphere turbulence on coupling between atmospheric regions, J. Geomag. Geoelectr., 43 (Suppl.), 621-636, 1991.

Hocking, W. K., Dynamical coupling processes between the middle atmosphere and lower ionosphere, J. Atmos. Terr. Phys., 58, 735-752, 1996.

Hocking, W. K., System design, signal processing procedures and preliminary results for CLOVAR, The University of Western Ontario VHF Atmospheric Radar, Radio Sci., in press, 1997.

Hocking, W. K., and A. M. Hamza, A quantitative measure of the degree of anisotropy of turbulence in terms of atmospheric parameters, with particular relevance to radar studies, J. Atmos. Terr. Phys., in press, 1997.

Hocking, W. K., and T. Thayaparan, Simultaneous and co-located observation of winds and tides by MF and Meteor radars over 
London, Canada, $\left(43^{\circ} \mathrm{N}, 81^{\circ} \mathrm{W}\right)$ during 1994-1996, Radio Sci., in press, 1997.

Hocking, W. K., P. T. May, and J. Röttger, Interpretation, reliability and accuracies of parameters deduced by the spacedantenna method in middle atmosphere applications, Pure Appl. Geophys., 130, 571-604, 1989.

Hocking, W. K., S. Fukao, T. Tsuda, M. Yamamoto, T. Sato, and S. Kato, Aspect sensitivity of stratospheric VHF radiowave scatterers, particularly above $15 \mathrm{~km}$ altitude, Radio Sci., 25, 613-627, 1990.

Hocking, W. K., S. Fukao, M. Yamamoto, T. Tsuda, and S. Kato, Viscosity waves and thermal-conduction waves as a cause of 'specular' reflectors in radar studies of the atmosphere, Radio Sci., 26, 1281-1303, 1991.

Hoppe, U.-P., and D. C. Fritts, On the downward bias in vertical velocity measurements by VHF radars, Geophys. Res. Lett., 22, 619-622, 1995.

Kudeki, E., P. K. Rastogi, and F. Surucu, Systematic errors in radar wind estimation: implications for comparative measurements, Radio Sci., 28, 169-179, 1993.

Larsen, M. F., and J. Röttger, VHF and UHF Doppler radars as tools for synoptic research, Bull. Am. Meteorol. Soc., 63, 996$1008,1982$.

Larsen, M. F., and J. Röttger, VHF radar measurements of inbeam incidence angles and associated vertical-beam radial velocity corrections, J. Atmos Oceanic Technol., 8, 477-490, 1991.

Larsen, M. F., R. D. Palmer, S. Fukao, R. F. Woodman, M. Yamamoto, T. Tsuda, and S. Kato, An analysis technique for deriving vector winds and in-beam incidence angles from radar interferometer measurements, J. Atmos. Oceanic Technol., 9, 314, 1992.

Lesicar, D., and W. K. Hocking, Studies of seasonal behaviour of the shape of mesospheric scatterers using a $1.98-\mathrm{MHz}$ radar, $J$. Atmos. Terr. Phys., 54, 295-309, 1992.

Lesicar, D., W. K. Hocking, and R. A. Vincent, Comparative studies of scatterers observed by MF radars in the southern hemisphere mesosphere, J. Atmos. Terr. Phys., 56, 581-591, 1994.

Luce, H., M. Crochet, F. Dalaudier, C. Sidi, Interpretation of VHF ST radar vertical echoes from in situ temperature sheet observation, Radio Sci., 30, 1003-1025, 1995.

Mathews, J. D., Incoherent-scatter radar studies of the mesosphere, Handb. MAP, 13, 135-154, 1984.

May, P. T., S. Fukao, T. Tsuda, T. Sato, and S. Kato, The effect of thin scattering layers on the determination of wind by Doppler radars, Radio Sci., 23, 83-94, 1988.

McLandress, C., G. G. Shepherd, B. H. Solheim, M. D. Burrage, P. B. Hays, and W. R. Skinner, Combined mesosphere/thermosphere winds using WINDII and HRDI data from the Upper Atmosphere Research Satellite, J. Geophys. Res., 101, 1044110454, 1996.

Namboothiri, S. P., A. H. Manson, and C. E. Meek, E-region real heights and their implications for MF radar-derived wind and tidal climatologies, Radio Sci., 28, 187-202, 1993.

Nastrom, G. D., and T. E. Van Zandt, Mean Vertical Motions seen by Radar Wind Profilers, J. Appl. Meterorol., 33, 984-995, 1994.

Nastrom, G. D., and T. E. Van Zandt, Biases due to gravity waves in wind profiler measurements of winds, J. Appl. Meteorol., 35, 243-257, 1996.

Phillips, A., A. H. Manson, C. E. Meek, and E. J. Llewellyn, A long-term comparison of middle-atmosphere winds measured at Saskatoon $\left(52^{\circ} \mathrm{N}, 107^{\circ} \mathrm{W}\right)$ by a medium-frequency radar and a Fabry-Perot interferometer, J. Geophys. Res., 99, 12923-12936, 1994.

Röttger, J., Investigations of lower and middle atmosphere dynamics with spaced-antenna drift radars, J. Atmos. Terr. Phys., 43, 277-292, 1981.
Röttger, J., and H. M. Ierkic, Postset beam steering and interferometer applications of VHF radars to study winds, waves, and turbulence in the lower and middle atmosphere, Radio Sci., 20, 1461-1480, 1985.

Röttger, J., and R. A. Vincent, VHF radar studies of tropospheric velocities and irregularities using spaced-antenna techniques, Geophys. Res. Lett., 5, 917-920, 1978.

Röttger, J., J. Klostermeyer, P. Czechowsky, R. Ruester, and G. Schmidt, Remote sensing of the atmosphere by VHF radar experiments, Naturwissenschaften, 65, 285-296, 1978.

Röttger, J., C. H. Liu, J. K. Chao, A. J. Chen, C. J. Pan, and I. J. Fu, Spatial interferometer measurements with the Chung-Li VHF radar, Radio Sci., 25, 503-515, 1990.

Royrvik, O., Spaced-antenna drift at Jicamarca, mesospheric measurements, Radio Sci., 18, 461-476, 1983.

Shepherd, G. G., G. Thuillir, W. A. Gault, B. H. Solheim, C. Hersom, J. M. Alunni, J.-F. Brun, S. Brune, P. Charlot, L. L. Cogger, D.-L. Desaulniers, W. F. J. Evans, R. L. Gattinger, F. Girod, D. Harvie, R. H. Hum, D. J. W. Kendall, E. J. Llewellyn, R. P. Lower, J. Ohrt, F. Pasternak, O. Peillet, I. Powell, Y. Rochon, W. E. Ward, R. H. Wiens, and J. Wimperis, WINDII, the wind imaging interferometer on the upper- atmosphere research satellite, J. Geophys. Res., 98, 10725-10750, 1993.

Sica, R. J., and M. D. Thorsley, Measurements of superadiabatic lapse rates in the middle atmosphere, Geophys. Res. Lett., 23, 2797-2800, 1996.

Stubbs, T. J., The measurement of winds in the D-region of the ionosphere by the use of partially reflected radio waves, $J$. Atmos. Terr. Phys., 35, 909-919, 1973.

Stubbs, T. J., and R. A. Vincent, Studies of D-region drifts during the winters of 1970-72, Aust. J. Phys., 26, 645-660, 1973.

Surucu, F., S. J. Franke, and E. Kudeki, On the influence of specular reflections in MF radar wind measurements, Radio Sci., 30, 1229-1244, 1995.

Tsuda, T., T. Sato, K. Hirose, S. Fukao, and S. Kato, MU radar observations of the aspect sensitivity of backscattered VHF echo power in the troposphere and lower stratosphere, Radio Sci., 21, 971-980, 1986.

Turek, R. S., K. L. Miller, R. G. Roper., and J. W. Brosnathan, Mesospheric wind studies during AIDA Act 89: morphology and comparison of various technique, J. Atmos. Terr. Phys., 57, 1321-1343, 1995.

Vincent, R. A., and T. J. Stubbs, A study of motions in the winter mesosphere using the partial reflection drift technique, Planet. Space Sci., 25, 441-455, 1977.

Vincent, R. A., T. J. Stubbs, R. H. O. Pearson, K. H. Lloyd, and C. H. Low, A comparison of partial reflection drifts with winds determined by rocket techniques - I, J. Atmos. Terr. Phys., 39, 813-821, 1977.

Vincent, R. A., P. May, W. K. Hocking, W. G. Elford, B. Candy, and B. H. Briggs, "First results with the Adelaide VHF radar: spaced-antenna studies of tropospheric winds, J. Atmos. Terr. Phys., 49, 353-366, 1987.

Weber, B. L., and D. B. Wuertz, Comparisons of rawinsonde and windprofiler radar measurements, J. Atmos. Oceanic Technol., 7, 157-174, 1990.

Woodman, R. F., Spectral moment estimation in MST radars, Radio Sci., 20, 1185-1195, 1985.

Woodman, R. F., and Y-H Chu, Aspect sensitivity measurements of VHF backscatter made with the Chung-LI radar: plausible mechanisms, Radio Sci., 24, 113-125, 1989.

Woodman, R. F., and A. Guillen, Radar observations of winds and turbulence in the stratosphere and mesosphere, J. Atmos. Sci., 31, 493-505, 1974.

Zrnic, D. S., Estimation of spectral moments for weather echoes, IEEE Trans. Geosci. Electron., GE-17, 113-128, 1979. 